\title{
Mitigating traceability risks amongst SMEs along the beef supply chain: A multiple case study approach to investigating the role and potential impact of information technology
}

\section{Mazino Amuno}

eLogistics Research Group

School of Technology, Engineering \& Design

University of Tasmania

Hobart, Australia

Email: mazino.amuno@utas.edu.au

\section{Mohammad Sadegh Taskhiri}

eLogistics Research Group

School of Technology, Engineering \& Design

University of Tasmania

Hobart, Australia

Email: mohammadsadegh.taskhiri@utas.edu.au

\section{Paul Turner}

eLogistics Research Group

School of Technology, Engineering \& Design

University of Tasmania

Hobart, Australia

Email: paul.turner@utas.edu.au

\begin{abstract}
This research-in-progress investigates how information technology can be used to mitigate increasing traceability risks being faced by small and medium sized enterprises (SMEs) along beef industry supply chains. Changing consumer preferences for information on product provenance as well as enhanced food safety requirements have increased the need for businesses to improve their traceability. Unfortunately, most traceability system are designed for large organisations in well-integrated supply chains. For SMEs in fragmented food chains finding ways to respond has proven difficult. Deploying a heuristic traceability framework, the research has used a multiple case study approach that has involved the development, implementation and current on-going evaluation of some low-cost IT traceability tools in 5 different beef supply chain segments involving SMEs. Preliminary results provide new perspectives on the role and potential impact of low cost IT for mitigating risks amongst SMEs in beef chains and for further refining the heuristic traceability framework for SMEs.
\end{abstract}

Keywords Traceability, beef chain, SMEs, Information Technology, risks, case study 


\section{Introduction}

Small and medium sized enterprises (SMEs) in the beef industry usually operate in complex multi-tiered networks involving numerous stakeholders along beef production and consumption value chains. This complexity often is accompanied by fragmentation that inhibits co-ordination between participants and the integration of information and product flows across various organisations(Adam et al. 2016). SMEs in the beef industry also display relatively low levels of technology use and technology sophistication across functional segments of their chains with paper documentation still very common. In this context, it is perhaps not surprising that SMEs ability to respond to traceability risks and challenges remains relatively low (Brooks et al. 2017).

The traceability risks and challenges increasingly being faced by SMEs relate to: (a) Provenance: retention of identity from the time an animal is born to when it is presented to the consumer as a cut of meat; (b) Food safety: whereby the consumer must be able to eat the meat without fear of adverse health effects, and, (c) Authenticity: provides consumers' with confidence that the meat is in fact what it purports to be on the label (Shackell 2008) (d) Animal Welfare: ascertaining and assuring consumers that the animals have been treated humanely on farm, during transportation, and prior to slaughter (Vanhonacker et al., 2007).

In responding to these challenges the research literature provides evidence that information technology (IT) adoption and use at different points in the supply chain can support greater awareness and visibility and potentially lead to effective risk mitigation (Cipolat 2016). Examples of IT use in addressing traceability issues include the use of radio frequency for identification (RFID) technologies and wireless sensor networks (WSN)(Carthy and Villalba 2018), and intelligent sensing and smart packaging systems (Sohail et al. 2018). More recently emerging technologies such as block-chains and distributed ledger technologies have been utilised to improve visibility and transparency of supply chains (Benton et al. 2018). However, considerable research has also highlighted that, while large organisations have been able to succcessfully implement these technologies in their supply chains, most SMEs continue to experience difficulties (Galliano and Orozco 2008). This situation for SMEs is in part due to a limited understanding and prioritisation of IT as a critical part of responding to the very real risks being faced and partly due to limited resources (Mattevi and Jones 2016). From a research perspective, it can also be argued that this lack of IT adoption and use is also linked to the reality that most of the models, tools, and frameworks advocated for enhancing traceability have been developed primarily with large businesses in highly integrated supply chains. As low SME adoption of these traceability approaches illustrate, most have proven to be inappropriate, impractical and/or too expensive for SMEs to adopt and use. As a result, SMEs have tended to only focus on minimum traceability in terms of tracking or tracing their products with immediate industry partners in what has been called a one-up-one-down (OUOD) siloed strategy (Nishantha et al. 2010). Unfortunately, with changing consumer preferences and enhanced food safety regulations this traceability approach is increasingly inadequate for SMEs to respond to the very real risks and uncertainties they are facing along beef supply chains.

This research-in-progress investigates how information technology can be used to mitigate traceability risks including provenance, meat safety, meat quality/authenticity, and animal welfare. being faced by SMEs along beef industry supply chains. The main contribution of this paper involves the development of a heuristic framework for IT traceability amongst SMEs that extends the work of Caridi et al. (2010) and adapts it to the specific case of developing an alternative approach for mitigating traceability risks amongst SMEs in beef chains. To further validate this framework and to increase detailed understanding of the role and potential impact of IT the research describes a multiple case study approach that has involved the development, implementation and current on-going evaluation of some low-cost IT traceability tools in 5 different beef supply chain segments.

\section{A Heuristic Framework for IT Traceability in SMEs}

In the last decade, understanding how to improve the visibility and alignment of information and product flows (traceability) amongst SMEs in food supply chains has emerged as an area of increasing importance. Approaches have included the use of mathematical models, simulation, and analytical modelling techniques (Dupuy et al. 2005) and the use of conceptual frameworks and reference models to harmonise and standardise data along food chains (Zhang and Bhatt 2014). These models and frameworks, whilst helpful, assume linear supply chains that are vertically integrated and, in most cases, involve primarily large businesses. For SMEs limited visibility and alignment of information and product flows beyond immediate supply chain partners has remained a problem and is not effectively 
addressed in these widely cited models/frameworks. In this context, Caridi et al. (2010) proposed a systematic and structured approach that might be suitable for adaptation to SMEs and for use in more fragmented supply chains. In their approach, visibility is measured according to the amount and quality of useful information when compared to the total information that could be exchanged between nodes in a supply chain (Nguyen et al. 2017). This approach also opens up the possibility of better analysing the potential impact of visibility improvement on the supply chains using IT. However, this does assume that adequate baseline data on total information and information quality can be captured. Building on the work of Caridi et al. (2010), this research has developed a heuristic framework for guiding the conduct of a number of field studies at different points along SME beef chains.

In this framework supply chain visibility is determined by the amount and quality of information that an organisation can readily access and views at each node along the supply chain. The heuristic framework uses three information quality metrics: (a) Freshness: the degree of information "synchronisation" with business partners; (b) Accuracy: the degree of conformity of the shared information with its actual value; (c) Completeness: the degree of completeness of shared information. From among 22 metrics identified in a review of literature on evaluating information quality status in organisations e.g. assessment methods for information quality (AIMQ) criteria (Naumann and Rolker 2005), the three metrics adopted were found to be the most significant in terms of measuring visibility in multi-tiered supply chains (Caridi et al. 2013). Based on these metrics, it is possible to evaluate supply chain visibility as being the sum of visibility of information that any specific company has access to and views at different nodes/segments in a supply chain. In conducting the field studies this research has used four types of traceability information flows to focus on across the different segments of the beef chain. The four types of traceability information are: $s=$ safety, $q=$ quality, $p=$ provenance and $w=$ animal welfare. These four traceability information are indicative of the major risks factors impacting beef supply chains at different segments(Shackell 2008). Therefore in evaluating traceability information flows = " $t$ " the approach is to use the formula $t=(s, q, p, w)$. For each type of traceability information flow "t", the following definitions are used:

1. Meat Safety: Information related to the chemical, microbiological or physical attributes of food products;

2. Meat quality: Information describing the compositional quality (lean to fat ratio, meat percentage, intramuscular fat, marbling, protein, and muscle area); functional quality ( e.g. $\mathrm{pH}$, and cooking loss); and eating quality or palatability of meat (e.g. appearance, juiciness, tenderness, and flavor)(ElMasry and Sun 2010);

3. Animal welfare: Information that describes the welfare status of an animal along the supply chain. Information includes the treatment animals receive e.g. animal care, animal husbandry, and humane treatment during transport and slaughter; and

4. Provenance: Information describing the origin, history and location of a product along the supply chain e.g. geography, region, or country of origin

Using the three information quality metrics provided for defining visibility (freshness; accuracy; amount/quantity) Table I illustrates how assessment calculations for each type of traceability information are being generated and how a visibility index is being calculated at each supply node for: $c=$ completeness (quantity); a=accuracy; and, $f=$ freshness. Where completeness is described as the quantity of information, and freshness and accuracy are defined in terms of the quality of information. In implementing this framework in the case studies, participants are requested to use a four-point rating scale to conduct a self-assessment of the visibility score for each type of traceability information (1-low to 4-high). This self-assessment approach provides quantitative data on comparative judgements on information quality status in organisations and along the supply chain because it takes into consideration the perspective of information sources, information users, and their information query processes (Naumann and Rolker 2005).

\begin{tabular}{lllll}
\hline & Safety (s) & Quality(q) & Provenance(p) & Animal welfare(w) \\
\hline Completeness(Quantity) & $t_{c, s}$ & $t_{c, q}$ & $t_{c, p}$ & $t_{c, w}$ \\
Accuracy & $t_{a, s}$ & $t_{a, q}$ & $t_{a, p}$ & $t_{a, w}$ \\
Freshness & $t_{t, s}$ & $t_{f, q}$ & $t_{f, p}$ & $t_{f, w}$ \\
\hline
\end{tabular}

Table 1. Assessments of supply visibility of traceability information (adapted from Caridi et al. (2010))

Table 2, illustrates the assessments used for each of the information quality criteria presented in relation to the four types of information flows. The table shows these assessments include completeness, 
accuracy, freshness, overall visibility for a given type of information, and quality of overall visible information.

\begin{tabular}{ll}
\hline Indicator & \multicolumn{1}{c}{ Formula } \\
\hline $\begin{array}{l}\text { Completeness/Quantity of overall visible } \\
\text { information }\end{array}$ & Node_visibility_completeness $s_{k}=\sqrt[4]{t_{c, s^{*}} t_{c, q^{*}} t_{c, p^{*}} t_{c, w}}$ \\
Accuracy of the overall visible information & Node_visibility_freshness $s_{k}=\sqrt[4]{t_{a, s^{*}}} t_{a, q^{*}} t_{a, p^{*}} t_{a, w}$ \\
Freshness of the overall visible information & Node_visibility_freshness ${ }_{k}=\sqrt[4]{t_{f, s^{*}}} t_{f, q^{*}} t_{f, p^{*}} t_{f, w}$ \\
$\begin{array}{l}\text { Overall visibility for a given type } i \\
\text { information at a node } k\end{array}$ & Node_partial_visibilityi,k $=\sqrt[3]{t_{c, s^{*}}} t_{a, s} t_{f, s}$ \\
Quality of the overall visible information & Node_visibility_quality ${ }_{k}=\sqrt[V]{ }$ \\
& $\begin{array}{l}\text { Node_visibility_accuracy } \\
\text { Node_visibility_freshness }\end{array}$
\end{tabular}

Table 2. Assessment of information quality metrics for visibility of information at each supply chain node (adapted from Caridi et al. (2010)

\subsection{Measuring visibility at individual supply chain segments}

Table 3 illustrates the nature of the scales that participants are asked to use in self-assessment of organisational visibility and traceability information related to meat safety, meat quality, provenance and animal welfare. A full taxonomic classification of measurable variables related to these four traceability risks factors can be found in Molnár et al. (2011). The criteria used for each are accessibility to information, quality of the information and information completeness respectively. For example, in the area of meat safety, temperature is considered an important metric especially in the cold chain from the processor to retail. For provenance, important indicators include geographical positional systems (GPS) readouts at handovers points along the chain, country of origin labelling, and regional location labelling, as well as ingredients percentage labelling. In the area of meat quality, the $\mathrm{pH}$ is also considered an important measure of the quality of meat (Mach et al. 2008). In the area of animal welfare, studies have found the usefulness of collecting accelerometer and inertia data to validate the welfare status of cattle (Robert et al. 2009).

\begin{tabular}{|c|c|c|c|c|c|}
\hline $\begin{array}{l}\text { Traceability } \\
\text { information } \\
\text { Freshness }\end{array}$ & $\begin{array}{l}\text { Supply } \\
\text { chain node } \\
\text { (o) }\end{array}$ & $\begin{array}{l}\text { I have access } \\
\text { to none or less } \\
\text { than } 25 \% \\
\text { information } \\
\text { (1) }\end{array}$ & $\begin{array}{l}\text { I have access } \\
\text { to at least } \\
\text { between } \\
25 \%-50 \% \\
\text { information } \\
\text { (2) }\end{array}$ & $\begin{array}{l}\text { I have } \\
\text { access to at } \\
\text { least 50- } \\
\text { than } 75 \% \\
\text { information } \\
\text { (3) }\end{array}$ & $\begin{array}{l}\text { I have access to at } \\
\text { least than } 75 \% \text { or } \\
\text { more information } \\
\text { (4) }\end{array}$ \\
\hline $\begin{array}{l}\text { Traceability } \\
\text { information } \\
\text { Accuracy }\end{array}$ & $\begin{array}{l}\text { Supply } \\
\text { chain node }\end{array}$ & $\begin{array}{l}\text { The accuracy } \\
\text { of exchanged } \\
\text { information is } \\
\text { usually very } \\
\text { low and } \\
\text { unsatisfactory } \\
\text { (1) }\end{array}$ & $\begin{array}{l}\text { The accuracy } \\
\text { of exchanged } \\
\text { information } \\
\text { is usually } \\
\text { satisfactory } \\
\text { but } \\
\text { situations in } \\
\text { which } \\
\text { information } \\
\text { is incorrect is } \\
\text { not u } \\
\text { common } \\
\text { (2) }\end{array}$ & $\begin{array}{l}\text { The } \\
\text { accuracy of } \\
\text { exchanged } \\
\text { information } \\
\text { is usually } \\
\text { satisfactory } \\
\text { which } \\
\text { information } \\
\text { in few } \\
\text { situations } \\
\text { (3) }\end{array}$ & $\begin{array}{l}\text { The accuracy of } \\
\text { exchanged } \\
\text { information is usually } \\
\text { satisfactory and very } \\
\text { accurate } \\
\text { (4) }\end{array}$ \\
\hline
\end{tabular}




\begin{tabular}{lll}
\hline Traceability & Supply & Information is \\
information & chain node & not always \\
Completeness & & updated and \\
(Quantity) & & not \\
& & satisfactory
\end{tabular}

(1)

Information
is only
updated
when I ask
suppliers to
provide data

(2)

In some
cases
information
is updated
when the
node is
asked to
provide
data

(3)

Table 3. Judgement scale of visibility metrics for each traceability information at each supply chain node (adapted from Caridi et al. (2010)

\section{Methodology}

The methodology being used in this research involves field studies with SMEs at 5 different beef supply chain segments in the Tasmanian beef industry. The choice of this multiple case study is appropriate for this study given that it follows replication rather than a sampling logic in the conduct of the research investigation (Noor 2008). This also provides an opportunity to identify and explore patterns between cases in order to validate the applicability of the proposed heuristic framework. The research strategy is organised in three phases of pre-intervention, intervention and post-intervention. The pre-intervention phase is organised into two steps. Firstly, preliminary engagement with 6 industry stakeholders in the Tasmanian beef chain was conducted to understand and validate whether these four major traceability risks are impacting the chain and to determine important metrics and indicators that can be used to characterize the risks. These industry stakeholders were selected from six major departments responsible for assessing and mitigating traceability risks at different segments, namely: Biosecurity and Traceability, Animal Brands, Meat Safety, Animal Welfare, Agricultural Veterinary (Agvet) and Chemicals, and Food Standards and local council food retail inspection. These are the major traceability risks areas impacting Tasmanian SMEs along the beef chain. Secondly, baseline data was collected from 8 participants from different beef supply chain segments within the Tasmanian beef industry who, following ethics approval, were invited via telephone to participate in face-to-face interactions across the three stages of the research. In phase one, the aim was to map the supply chain and to benchmark each participants' level of visibility at each segment in relation to meat safety, meat quality/authenticity, provenance, and animal welfare. The participants were drawn from the following segments: farmer, saleyard, processor, cold chain transport, and retail butchers. Participants provided suggestions on the metrics which they considered useful for their supply chain operations.

In Phase 2 (the intervention phase) the research team developed a mobile application and implemented wireless time and temperature sensors in the beef chain to investigate their impact in improving the quality of information available and perceptions of stakeholders around how this information could be used to increase visibility and potentially mitigate traceability risks in their supply chain segment. In phase 3 current on-going evaluation is occurring using both quantitative and qualitative techniques. It is anticipated as well as generating results on how these low-cost IT solutions have impacted on different SMEs along the beef chain, it will also be possible to further refine the heuristic framework that guided the approach to the multiple case studies.

At the level of the research design a mixed mode of data collection using semi-structured interviews, questionnaires were deployed. This allowed the development of a baseline map of the beef chain. Each participant was interviewed for approximately 60-90mins and additional documents related to interview responses were obtained for triangulation purposes and to improve the reliability of findings. Data analysis was conducted using qualitative and quantitative techniques. Voice transcripts derived from interviews were coded and analysed thematically to generate key themes emerging from the data. Paper documents derived from field investigation were analysed using both document and context analysis procedures. The questionnaires were analysed and interpreted using the assessment formulae presented in the tables above. This quantitative self-assessment is allowing for comparison with participants in Phase 3 evaluation. In the next section, preliminary results from case 1 involving three retail meat butcher stores are presented. The results highlight their current levels of visibility to traceability risks of safety, provenance, quality, and animal welfare in the beef chain. 


\section{Preliminary Results and Next Steps}

This section presents preliminary findings from one segment of the beef chain involving three retail butcher stores in Tasmania. The results from phase 1 involving three retail butchers confirmed that across the cases the level of traceability and use of technology was low. In one case, there were significant traceability issues related to accuracy of provenance information. One butcher commented: " $I$ don't market my beef as "xyz" or anything like that. I market my chicken as "abc" cos I buy my chicken from "abc". I buy my pork from "efg" and I market those as such. But our beef, i'll just.. if customer asks, i'll give them a fair and honest answer. I can know where my products coming from generally but not a $100 \%$ of the time"-Butcher A. This butcher explained that in many cases, it is difficult to market beef as Tasmanian beef due to poor traceability and also due to further disaggregation and mixing that occurs in the store. As a result, they prefer not to differentiate their local beef due to prevalent identity preservation challenges.

In the area of meat quality, authenticity and animal welfare were identified as key traceability challenges in Butcher B. The butcher commented about the issue of freshness and completeness of information as follows: "Consumers sometimes ask where the products are from? Whether they are free-range, what type of breed, how it was treated, how old it is, specific cut, cattle sex, and whether it is left or right leg. Sometimes they ask which part of the body it is from. if they ask, we tell them primarily verbally "-Butcher $B$. The butcher described a key problem related to gaining access to information in real time. In most cases, a follow-up phone call conversation with processors is required to access more information, and rarely do they have that information in real time when consumers demand for better information in the store.

Regarding the issue meat safety and integrity, Butcher $\mathrm{C}$ showed a marked difference as compared to Butcher A and B. There was limited interest in verifying meat integrity and safety in the cold chain. Trust and relationship quality were observed as significant predictors of perception bias despite the lack of any technologies. The response is as follows: . No I don't check what temperature it comes since they have a log and they just look at it and tell you when its dropped. Not with "ggf company" because I believe they are a professional operators, and as soon as there is a problem. They get another truck...as I said. They have things in place"-Butcher C

In phase 2, a number of low-cost technologies were developed and implemented to improve visibility and enhance traceability in the case study, and they include: (a) 3 mobile applications (iOS and Android) integrated with quick response code and near field communication (NFC) reading functionality to improve consumer access and timeliness of traceability information related to beef provenance and meat safety in the 3 retail butcher stores; (b) 1 meat integrity android application, called chill verify, to improve completeness/accessibility to time and temperature readings in the cold chain between processor, cold transport, and retail butchers; (c) 2 low cost wireless temperature monitoring sensors to improve freshness and accuracy of equipment temperature in retail butcher stores; (d) 1 consumer verification app integrated with smart meat labels to verify complete information related to animal welfare, meat quality, province and meat safety directly on meat packages; and (e) 2 wireless activity monitoring sensors have been purchased and will be tested in the field to improve accuracy and accessibility to more animal activity data in the farm and during transport.

Preliminary evaluation is revealing markedly different reactions within and between different nodes. For example, amongst butchers there have been some who have enthusiastically adopted and use the trial systems and who are now seeking to invest and implement them into the future. Other butchers however have struggled and continue not to see value in improving their supply chain visibility and capacity for traceability. In reflecting on the heuristic framework, early indications are that it is helpful for obtaining quantitative judgement on visibility from a focal company's point of view, however to eliminate bias in judgement, it is important that multiple sources of data be utilised. This is because, key observations from the field show that contextual factors such as trust and relationship quality could potentially lead to judgement bias. Thus, mixed methods evaluation triangulating interview responses with quantitative judgement from questionnaires were utilised to address this short coming. This study has contributed to the development of a framework for IT traceability in SMEs. This framework provides an alternative approach for SMEs to enhance visibility of alignment of information and material flow, and potentially mitigate traceability risks using low-cost IT tools. The next steps are to finalise the evaluation phase and complete this doctoral research. 


\section{References}

Adam, B. D., Holcomb, R., Buser, M., Mayfield, B., Thomas, J., O’Bryan, C. A., Crandall, P., Knipe, D., Knipe, R., and Ricke, S. C. 2016. "Enhancing Food Safety, Product Quality, and Value-Added in Food Supply Chains Using Whole-Chain Traceability,").

Benton, M. C., Radziwill, N., Purritano, A., and Gerhart, C. 2018. "Blockchain for Supply Chain: Improving Transparency and Efficiency Simultaneously," Software Quality Professional (20:3).

Brooks, S., Elliott, C. T., Spence, M., Walsh, C., and Dean, M. 2017. "Four Years Post-Horsegate: An Update of Measures and Actions Put in Place Following the Horsemeat Incident of 2013," npj Science of Food (1:1), p. 5 .

Caridi, M., Crippa, L., Perego, A., Sianesi, A., and Tumino, A. 2010. "Measuring Visibility to Improve Supply Chain Performance: A Quantitative Approach," Benchmarking: An International Journal (17:4), pp. 593-615.

Caridi, M., Perego, A., and Tumino, A. 2013. "Measuring Supply Chain Visibility in the Apparel Industry," Benchmarking: An International Journal (20:1), pp. 25-44.

Carthy, M., and Villalba, R. 2018. "New Trends in Cold Chain Monitoring Applications-a Review," Food control).

Cipolat, A. C. 2016. "Supply Chain Risk Mitigation through Visibility and Collaboration for Smes in the South African Manufacturing Enviroment."

Dupuy, C., Botta-Genoulaz, V., and Guinet, A. 2005. "Batch Dispersion Model to Optimise Traceability in Food Industry," Journal of Food Engineering (70:3), pp. 333-339.

ElMasry, G., and Sun, D.-W. 2010. "Meat Quality Assessment Using a Hyperspectral Imaging System," in Hyperspectral Imaging for Food Quality Analysis and Control. Elsevier, pp. 175-240.

Galliano, D., and Orozco, L. 2008. "Intra and Inter Organisational Determinants of Electronic-Based Traceability Adoption: Evidences from the French Agri-Food Industry," 12th Congress of the European Association of Agriculture Economics, Ghent, Belgium.

Mach, N., Bach, A., Velarde, A., and Devant, M. 2008. "Association between Animal, Transportation, Slaughterhouse Practices, and Meat Ph in Beef," Meat Science (78:3), pp. 232-238.

Mattevi, M., and Jones, J. A. 2016. "Traceability in the Food Supply Chain: Awareness and Attitudes of Uk Small and Medium-Sized Enterprises," Food Control (64), pp. 120-127.

Molnár, A., Van Lembergen, K., Gellynck, X., Sebok, A., and Berczeli, A. 2011. "What Can We Learn from Best Practices Regarding Food Chain Transparency?," Proceedings in Food System Dynamics), pp. 435-446.

Naumann, F., and Rolker, C. 2005. Assessment Methods for Information Quality Criteria. HumboldtUniversität zu Berlin, Mathematisch-Naturwissenschaftliche Fakultät II, Institut für Informatik.

Nguyen, H. V., Nguyen, H. T., Deligonul, S., and Cavusgil, S. T. 2017. "Developing Visibility to Mitigate Supplier Risk: The Role of Power-Dependence Structure," Asia-Pacific Journal of Business Administration (9:1), pp. 69-82.

Nishantha, G., Wanniarachchige, M., and Jehan, S. 2010. "A Pragmatic Approach to Traceability in Food Supply Chains," Advanced Communication Technology (ICACT), 2010 The 12th International Conference on: IEEE, pp. 1445-1450.

Noor, K. B. M. 2008. "Case Study: A Strategic Research Methodology," American journal of applied sciences (5:11), pp. 1602-1604.

Robert, B., White, B., Renter, D., and Larson, R. 2009. "Evaluation of Three-Dimensional Accelerometers to Monitor and Classify Behavior Patterns in Cattle," Computers and Electronics in Agriculture (67:1-2), pp. 80-84.

Shackell, G. H. 2008. "Traceability in the Meat Industry-the Farm to Plate Continuum," International journal of food science \& technology (43:12), pp. 2134-2142.

Sohail, M., Sun, D.-W., and Zhu, Z. 2018. "Recent Developments in Intelligent Packaging for Enhancing Food Quality and Safety," Critical reviews in food science and nutrition), pp. 1-13.

Zhang, J., and Bhatt, T. 2014. "A Guidance Document on the Best Practices in Food Traceability," Comprehensive Reviews in Food Science and Food Safety (13:5), pp. 1074-1103.

\section{Acknowledgements}

The authors acknowledge the support of the Australian Research Council Industrial Transformation Research Hub 'Pathways to Market' http://www.utas.edu.au/pathways-to-market 
Copyright: (C) 2018 authors. This is an open-access article distributed under the terms of the Creative Commons Attribution-NonCommercial 3.0 Australia License, which permits non-commercial use, distribution, and reproduction in any medium, provided the original author and ACIS are credited. 